\title{
A new characterization of Conrad's property for group orderings, with applications
}

\author{
ANDRÉS NAVAS \\ CRISTÓBAL RIVAS \\ APPENDIX BY ADAM CLAY
}

\begin{abstract}
We provide a pure algebraic version of the first-named author's dynamical characterization of the Conrad property for group orderings. This approach allows dealing with general group actions on totally ordered spaces. As an application, we give a new and somehow constructive proof of a theorem first established by Linnell: an orderable group having infinitely many orderings has uncountably many. This proof is achieved by extending to uncountable orderable groups a result about orderings which may be approximated by their conjugates. This last result is illustrated by an example of an exotic ordering on the free group given by the third author in the Appendix.
\end{abstract}

06F15, 20F60; 57S25

\section{Introduction}

In recent years, relevant progress has been made in the theory of (left) orderable groups. This has been achieved mainly by means of the use of a recently introduced mathematical object, namely the space of group orderings (see for instance Clay [2], Linnell [9], Morris [11], Navas [13] and Sikora [14]). This space may be endowed with a natural topology (roughly, two orderings are close if they coincide over large finite sets), and the study of this topological structure should reveal some algebraic features of the underlying group. In [13] it was realized that, for this study, the classical Conrad property for group orderings becomes relevant. Bringing ideas and techniques from the theory of codimension-one foliations, the 'dynamical' insight of this property was revealed. Unfortunately, many proofs of [13] are difficult to read for people with a pure algebraic view of orderable groups. More importantly, some of the results therein do not cover the case of uncountable groups. Indeed, the dynamical analysis of group orderings is done via the so-called 'dynamical realization' of orderable groups as groups of homeomorphisms of the line, which is not available for general uncountable orderable groups. 
Motivated by this, we develop here an algebraic counterpart of (part of) the analysis of [13]. We begin by giving a new characterization of the Conrad property that is purely algebraic, although it has a dynamical flavor (see Theorem 2.4). This leads naturally to the notion of Conradian actions on totally ordered spaces. A relevant example concerns the action of an ordered group on the space of cosets with respect to a convex subgroup. In this setting, we define the notion of Conradian extension (see Example 2.11), and we generalize Conrad's classical theorem on the 'level' structure of groups admitting Conradian orderings (see Theorem 2.14, Corollary 2.15).

A relevant concept introduced in [13] is the Conradian soul, which corresponds to the maximal subgroup of an ordered group that is convex and restricted to which the ordering is Conradian. In [13], a more geometrical view of this notion was given in the case of countable groups. Here we provide an analogous algebraic description which applies to general (possibly uncountable) ordered groups (see Theorem 3.1).

The Conradian soul was introduced as a main tool for dealing with the problem of approximating a group ordering by its conjugates. For instance, it was shown in [13] that if the Conradian soul of an ordering on a non-trivial countable group is trivial, then this ordering is an accumulation point of its set of conjugates. The extension of this result to uncountable orderable groups appears here as Theorem 3.7. We point out that an independent proof using completely different ideas was given by Adam Clay in [2].

Based on the work of Linnell [9], it was shown in [13] that if an ordering on a group is isolated in the corresponding space of orderings, then its Conradian soul is 'almost trivial', in the sense that it has only finitely many orderings. It is then natural to deal with ordered groups $(\Gamma, \preceq)$ for which the Conradian soul $C_{\preceq}(\Gamma)$ is non-trivial but has only finitely many orderings. If $\preceq$ is not Conradian, then to each of the orderings on $C_{\preceq}(\Gamma)$ corresponds an ordering on $\Gamma$ (roughly, the new orderings on $\Gamma$ are obtained by changing the original one on $C_{\preceq}(\Gamma)$ but preserving the set of elements bigger than the identity outside). As it was proved in [13], at least one of these orderings on $\Gamma$ is an accumulation point of its set of conjugates provided that $\Gamma$ is countable. Here we extend this result to the case of uncountable groups (see Corollary 3.10).

The property of being approximated by its conjugates does not hold for all of the finitely many orderings on $\Gamma$ obtained by the preceding construction. A remarkable example illustrating this fact, namely the Dubrovina-Dubrovin ordering $\preceq_{D D}$ on braid groups $B_{n}$ [7], was extensively studied from this point of view in [13]. In the Appendix, Adam Clay provides a different kind of example, namely an 'exotic' ordering $\preceq_{C}$ on the free group $F_{2}$. As it is the case of $\preceq_{D D}$, the Conradian soul of $\preceq_{C}$ is isomorphic to $\mathbb{Z}$, and $\preceq_{C}$ is not an accumulation point of the set of its conjugates. (This answers by the negative a question suggested in [13, Remark 4.11].) The main difference between 
$\preceq_{D D}$ and $\preceq_{C}$ lies on the fact that $\preceq_{D D}$ is an isolated point of the (uncountable) space of orderings of $B_{n}$, while $\preceq_{C}$ is non-isolated in the (also uncountable) space of orderings of $F_{2}$. (Actually, the space of orderings of $F_{2}$ is homeomorphic to the Cantor set; see McCleary [10] and [13].)

As a final application of our methods, we give a new proof of a theorem first established by Linnell for countable groups [9]: if a group has infinitely many orderings, then it has uncountably many. Linnell's proof uses an argument from General Topology for reducing the general case to that of Conradian orderings for which prior arguments by Zenkov [15] apply. To deal with the non Conradian case, we use our machinery on Conradian souls. Note that this was already done in [13] for countable groups: Theorem 4.1 here corresponds to the extension to the case of uncountable groups.

\section{Acknowledgements}

The first author was funded by the PBCT/Conicyt Research Network on Low Dimensional Dynamics.

\section{Crossings and Conradian orderings}

\subsection{An equivalent for Conrad's property}

Let $\preceq$ be an ordering on a group $\Gamma$, that is, a total order relation which is invariant by left multiplication. Recall that $\preceq$ is said to be Conradian if for all $f \succ 1$ and all $g \succ 1$ (for short, for all positive elements $f, g$ ) there exists $n \in \mathbb{N}=\{1,2, \ldots\}$ such that $f g^{n} \succ g$. (See however Remark 2.5.) A subgroup $\Gamma_{0}$ of $\Gamma$ is $\preceq-$ Conradian if the restriction of $\preceq$ to it is a Conradian ordering.

A crossing for the ordered group $(\Gamma, \preceq)$ is a 5-tuple $(f, g, u, v, w)$ of elements in $\Gamma$ such that

- $u \prec w \prec v$,

- $g^{n} u \prec v$ and $f^{n} v \succ u$ for every $n \in \mathbb{N}$,

- there exist $M, N$ in $\mathbb{N}$ so that $f^{N} v \prec w \prec g^{M} u$.

Remark 2.1 It follows from the third condition that neither $f$ nor $g$ can be equal to the identity. 
Remark 2.2 If $(f, g, u, v, w)$ is a crossing, then the inequalities $f^{n} v \succ u$ and $g^{n} u \prec v$ actually hold for every integer $n$. Indeed, we necessarily have $f v \prec v$, since in the other case we would have $v \succ w \succ f^{N} v \succ f^{N-1} v \succ \cdots \succ f v \succ v$, which is absurd. Therefore, for $n>0$,

$$
f^{-n} v \succ f^{n-1} v \succ \cdots \succ f^{-1} v \succ v \succ u .
$$

The inequality $g^{-n} u \prec v$ for $n>0$ may be checked similarly.

Remark 2.3 The reason of the use of different type of letter for the elements $f, g$ and $u, v, w$ will become clear in Section 2.2. Somehow, $u, v, w$ should be thought of as 'reference points' instead of genuine group elements (see Figure 1).

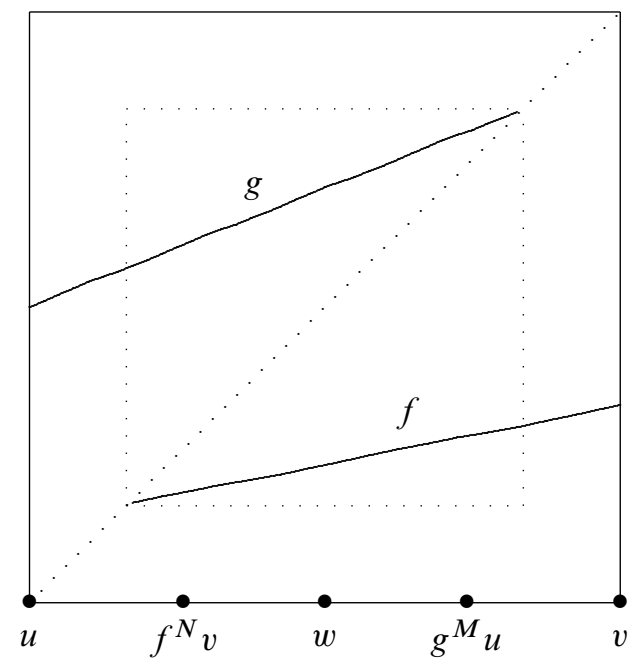

Figure 1: A crossing

The next result is the natural analogue of [13, Propositions 3.16 and 3.19] in our setting.

Theorem 2.4 The ordering $\preceq$ is Conradian if and only if ( $\Gamma, \preceq$ ) admits no crossing.

Proof Suppose that $\preceq$ is not Conradian, and let $f, g$ be positive elements so that $f g^{n} \prec g$ for every $n \in \mathbb{N}$. We claim that $(f, g, u, v, w)$ is a crossing for $(\Gamma, \preceq)$ for the choices $u=1, v=f^{-1} g$ and $w=g^{2}$. Indeed:

- From $f g^{2} \prec g$ one obtains $g^{2} \prec f^{-1} g$, and since $g \succ 1$, this gives $1 \prec g^{2} \prec$ $f^{-1} g$, that is, $u \prec w \prec v$. 
- From $f g^{n} \prec g$ one gets $g^{n} \prec f^{-1} g$, that is, $g^{n} u \prec v$ (for every $n \in \mathbb{N}$ ); on the other hand, since both $f, g$ are positive, we have $f^{n-1} g \succ 1$, and thus $f^{n}\left(f^{-1} g\right) \succ 1$, that is, $f^{n} v \succ u$ (for every $n \in \mathbb{N}$ ).

- The relation $f\left(f^{-1} g\right)=g \prec g^{2}$ may be read as $f^{N} v \prec w$ for $N=1$; on the other hand, the relation $g^{2} \prec g^{3}$ is $w \prec g^{M} u$ for $M=3$.

Conversely, assume that $(f, g, u, v, w)$ is a crossing for $(\Gamma, \preceq)$ so that $f^{N} v \prec w \prec$ $g^{M} u$ (with $M, N$ in $\mathbb{N}$ ). We will prove that $\preceq$ is not Conradian by showing that, for $h=g^{M} f^{N}$ and $\bar{h}=g^{M}$, both elements $w^{-1} h w$ and $w^{-1} \bar{h} w$ are positive, but

$$
\left(w^{-1} h w\right)\left(w^{-1} \bar{h} w\right)^{n} \prec w^{-1} \bar{h} w \quad \text { for all } n \in \mathbb{N} .
$$

To show this, first note that $g w \succ w$. Indeed, if not then we would have

$$
w \prec g^{N} u \prec g^{N} w \prec g^{N-1} w \prec \cdots \prec g w \prec w,
$$

which is absurd. Clearly, the inequality $g w \succ w$ implies

$$
g^{M} w \succ g^{M-1} w \succ \cdots \succ g w \succ w,
$$

and hence

$$
w^{-1} \bar{h} w=w^{-1} g^{M} w \succ 1
$$

Moreover,

$$
h w=g^{M} f^{N} w \succ g^{M} f^{N} f^{N} v=g^{M} f^{2 N} v \succ g^{M} u \succ w .
$$

and hence

$$
w^{-1} h w \succ 1
$$

Now note that, for every $n \in \mathbb{N}$,

$$
h \bar{h}^{n} w=h g^{M n} w \prec h g^{M n} g^{M} u=h g^{M n+M} u \prec h v=g^{M} f^{N} v \prec g^{M} w=\bar{h} w .
$$

After multiplying by the left by $w^{-1}$, the last inequality becomes

$$
\left(w^{-1} h w\right)\left(w^{-1} \bar{h} w\right)^{n}=w^{-1} h \bar{h}^{n} w \prec w^{-1} \bar{h} w,
$$

as we wanted to check. Together with (1) and (2), this shows that $\preceq$ is not Conradian. $\square$

Remark 2.5 A relevant fact that will be not used in this work is that, for every Conradian group ordering $\preceq$, one actually has $f g^{2} \succ g$ for all positive elements $f, g$ (that is, one can take ' $n=2$ ' in the original definition). The proof given in [13, Proposition 3.7] uses the fact that, if $f, g$ are positive elements for which $f g^{2} \prec g$, then letting $h=f g$ one has $f h^{n} \prec h$ for all $n \in \mathbb{N}$. This is illustrated by Figure 
2. Notice that, as shown below, in this situation $(f, f g, 1, f g, g)$ is a crossing for $M=N=2 \ldots$

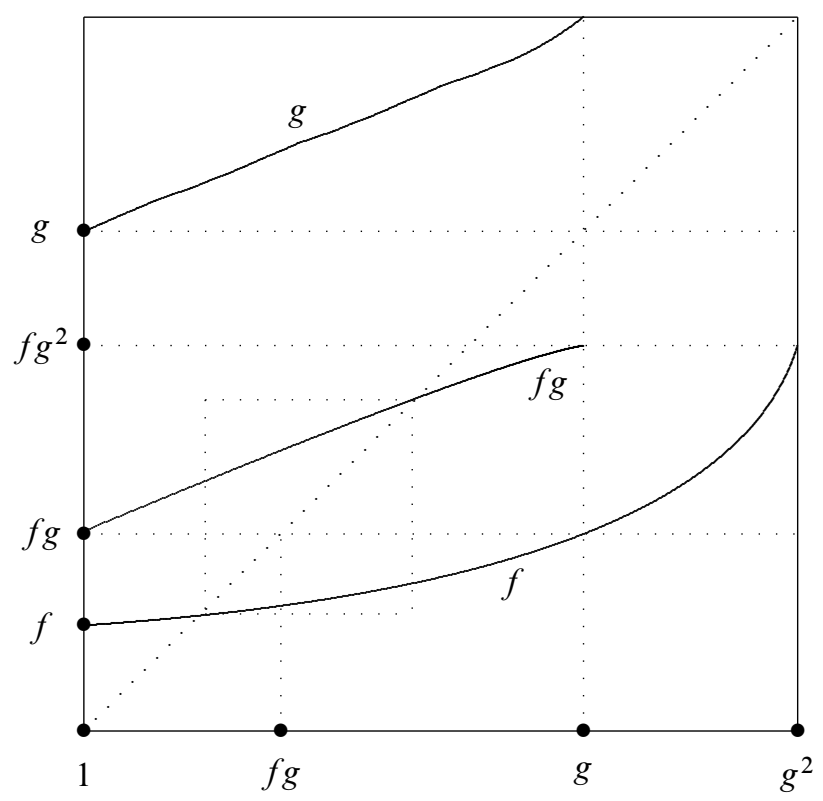

Figure 2: The ' $n=2$ ' condition

Remark 2.6 The second condition in the definition of crossing may seem difficult to handle. A more 'robust' property is that of reinforced crossing, which is a 5-tuple $(f, g, u, v, w)$ of elements in an ordered group $(\Gamma, \preceq)$ such that

- $u \prec w \prec v$,

- $f u \succ u$ and $g v \prec v$,

- there exist $M, N$ in $\mathbb{N}$ so that $f^{N} v \prec w \prec g^{M} u$.

One easily checks that a reinforced crossing is a crossing. Conversely, if $(f, g, u, v, w)$ is a crossing, then $\left(f^{N} g^{M}, g^{M} f^{N}, f^{N} w, g^{M} w, w\right)$ is a reinforced crossing (here, $M, N$ in $\mathbb{N}$ are such that $\left.f^{N} v \prec w \prec g^{M} u\right)$. Indeed, from the properties of crossing one gets $f^{N} g^{M}\left(g^{M} w\right) \prec f^{N} v \prec w$ and $g^{M} f^{N}\left(f^{N} w\right) \succ g^{M} u \succ w$. Moreover, $f^{N} g^{M}\left(f^{N} w\right) \succ f^{N} g^{M} u \succ f^{N} w$ and $g^{M} f^{N}\left(g^{M} w\right) \prec g^{M} f^{N} v \prec g^{M} w$.

Remark 2.7 Replacing $f$ (resp. $g$ ) by $f^{N}$ (resp. $g^{M}$ ) in the original definition, one readily checks that the condition of existence of a reinforced crossing is equivalent to the existence of a 5-tuple of group elements $(f, g, u, v, w)$ so that

$$
u \prec f u \prec f v \prec w \prec g u \prec g v \prec v .
$$




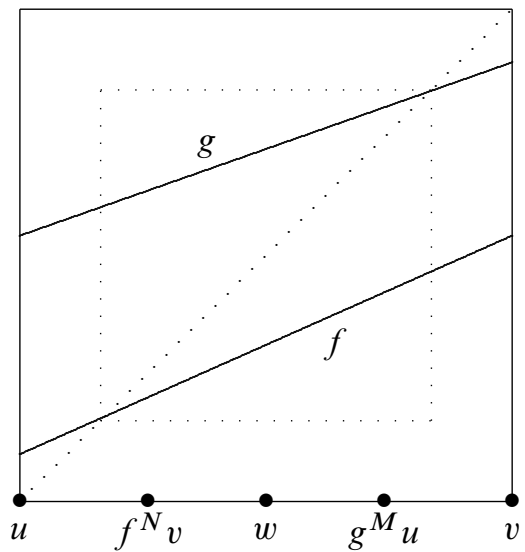

Figure 3: A reinforced crossing

With no doubt, this chain of inequalities should be easier to handle than the three conditions in Remark 2.6.

Remark 2.8 The dynamical characterization of Conrad's property may serve as inspiration for introducing other relevant properties for group orderings. (Compare [13, Question 3.22].) For instance, one can say that a 6-tuple $\left(f, g, u, v, w_{1}, w_{2}\right)$ of elements in an ordered group ( $\Gamma, \preceq)$ is a (reinforced) double crossing if (see Figure 4)

- $u \prec w_{1} \prec w_{2} \prec v$,

- $f u \succ u$ and $f v \succ v$,

- $g u \succ w_{1}, g v \prec w_{2}$, and $f w_{2} \prec w_{1}$.

Finding a simpler algebraic counterpart of the property of not having a double crossing for an ordering seems to be an interesting problem.

\subsection{An extension to group actions on ordered spaces}

Let $\Gamma$ be a group acting by order-preserving bijections on a totally ordered space $(\Omega, \leq)$. A crossing for the action of $\Gamma$ on $\Omega$ is a 5-tuple $(f, g, u, v, w)$, where $f, g$ belong to $\Gamma$ and $u, v, w$ are in $\Omega$, such that

- $u<w<v$,

- $g^{n} u<v$ and $f^{n} v>u$ for every $n \in \mathbb{N}$,

- there exist $M, N$ in $\mathbb{N}$ so that $f^{N} v<w<g^{M} u$. 


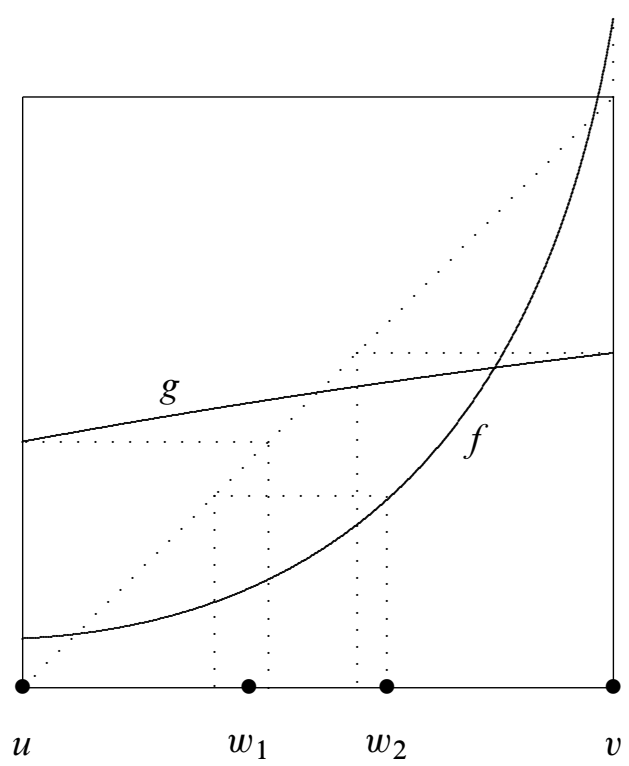

Figure 4: A (reinforced) double crossing

Example 2.9 The real line carries a natural total order, and thus our definition applies to groups acting on it by orientation preserving homeomorphisms. The notion of crossing for this case is exactly the same as that of elements in transversal position in [13, Definition 3.24].

Example 2.10 If $\Gamma$ is endowed with an ordering $\preceq$, one may take $(\Omega, \leq)=(\Gamma, \preceq)$ as a totally ordered set. The action of $\Gamma$ by left translations on it preserves the order: a crossing for this action corresponds to a crossing for $(\Gamma, \preceq)$, in the terminology of Section 2.1. Note that this example generalizes the preceding one for countable groups, since every countable ordered group may be canonically (up to semiconjugacy) realized as a group of orientation preserving homeomorphisms of the real line [13, Section 2.1].

For another relevant example recall that, given ordered group $(\Gamma, \preceq)$, a subset $S$ is $\preceq$-convex if for every $f_{1} \prec f_{2}$ in $S$, every $f \in \Gamma$ satisfying $f_{1} \prec f \prec f_{2}$ belongs to $S$. When $S$ is a subgroup, this is equivalent to that for all positive $\bar{f} \in S$, every $f \in \Gamma$ such that $1 \prec f \prec \bar{f}$ belongs to $S$.

Example 2.11 Let $(\Gamma, \preceq)$ be an ordered group, and let $\Gamma_{0}$ be a $\preceq$-convex subgroup. The space of left cosets $\Omega=\Gamma / \Gamma_{0}$ carries a natural total order $\leq$, namely $f \Gamma_{0}<g \Gamma_{0}$ if $f h_{1} \prec g h_{2}$ for some $h_{1}, h_{2}$ in $\Gamma_{0}$ (the reader will easily check that this definition is independent of the choice of $h_{1}$ and $h_{2}$ in $\Gamma_{0}$ ). The action of $\Gamma$ by left translations on 
$\Omega$ preserves this order. (Note that taking $\Gamma_{0}$ as being the trivial subgroup, this example reduces to the preceding one.) Whenever this action has no crossings, we will say that $\Gamma$ is a Conradian extension of $\Gamma_{0}$.

Remark 2.12 Let $(\Gamma, \preceq)$ be an ordered group, and let $\Gamma_{0}$ be a $\preceq-$ convex subgroup. Given any ordering $\preceq_{*}$ on $\Gamma_{0}$, the extension of $\preceq_{*}$ by $\preceq$ is the ordering $\preceq^{*}$ on $\Gamma$ for which $1 \prec^{*} f$ if and only if either $f \in \Gamma_{0}$ and $1 \prec_{*} f$, or $f \notin \Gamma_{0}$ and $1 \prec f$. The reader can easily check that $\Gamma_{0}$ is still a $\preceq^{*}$-convex subgroup of $\Gamma$. Moreover, $\Gamma$ is a $\preceq$-Conradian extension of $\Gamma_{0}$ if and only if it is a $\preceq^{*}$-Conradian extension of it.

For a general order-preserving action of a group $\Gamma$ on a totally ordered space $(\Omega, \leq)$, the action of an element $f \in \Gamma$ is said to be cofinal if for all $x<y$ in $\Omega$ there exists $n \in \mathbb{Z}$ such that $f^{n}(x)>y$. Note that if the action of $f$ is not cofinal, then there exist $x<y$ in $\Omega$ such that $f^{n}(x)<y$ for every integer $n$.

Proposition 2.13 Let $\Gamma$ be a group acting by order-preserving bijections on a totally ordered space $(\Omega, \leq)$. If the action of $\Gamma$ on $\Omega$ has no crossings, then the set of elements whose action is not cofinal forms a normal subgroup of $\Gamma$.

Proof Let us denote the set of elements whose action is not cofinal by $\Gamma_{0}$. This set is normal. Indeed, given $g \in \Gamma_{0}$, let $x<y$ in $\Omega$ be such that $g^{n}(x)<y$ for all $n$. For each $h \in \Gamma$ we have $g^{n} h^{-1}(h(x))<y$, and hence $\left(h g h^{-1}\right)^{n}(h(x))<h(y)$ (for all $n \in \mathbb{Z})$. Since $h(x)<h(y)$, this shows that $h g h^{-1}$ belongs to $\Gamma_{0}$.

It follows immediately from the definition that $\Gamma_{0}$ is stable under inversion, that is, $g^{-1}$ belongs to $\Gamma_{0}$ for all $g \in \Gamma_{0}$. The fact that $\Gamma_{0}$ is stable by multiplication is more subtle. For the proof, given $x \in \Omega$ and $g \in \Gamma_{0}$, we will denote by $I_{g}(x)$ the convex closure of the set $\left\{g^{n}(x): n \in \mathbb{Z}\right\}$, that is, the set formed by the $y \in \Omega$ for which there exists $m, n$ in $\mathbb{Z}$ so that $g^{m}(x) \leq y \leq g^{n}(x)$. Note that $I_{g}(x)=I_{g}\left(x^{\prime}\right)$ for all $x^{\prime} \in I_{g}(x)$; moreover, $I_{g^{-1}}(x)=I_{g}(x)$ for all $g \in \Gamma_{0}$ and all $x \in \Omega$; finally, if $g(x)=x$, then $I_{g}(x)=\{x\}$. We claim that if $I_{g}(x)$ and $I_{f}(y)$ are non-disjoint for some $x, y$ in $\Omega$ and $f, g$ in $\Gamma_{0}$, then one of them contains the other. Indeed, assume that there exist non-disjoint sets $I_{f}(y)$ and $I_{g}(x)$, none of which contains the other. Without loss of generality, we may assume that $I_{g}(x)$ contains points to the left of $I_{f}(y)$ (if this is not the case, just interchange the roles of $f$ and $g$ ). Changing $f$ and/or $g$ by their inverses if necessary, we may assume that $g(x)>x$ and $f(y)<y$, and hence $g\left(x^{\prime}\right)>x^{\prime}$ for all $x^{\prime} \in I_{g}(x)$, and $f\left(y^{\prime}\right)<y^{\prime}$ for all $y^{\prime} \in I_{y}(f)$. Take $u \in I_{g}(x) \backslash I_{f}(y), w \in I_{g}(x) \cap I_{f}(y)$, and $v \in I_{f}(y) \backslash I_{g}(x)$. Then one easily checks that $(f, g, u, v, w)$ is a crossing, which is a contradiction. 
Let now $g, h$ be elements in $\Gamma_{0}$, and let $x_{1}<y_{1}$ and $x_{2}<y_{2}$ be points in $\Omega$ such that $g^{n}\left(x_{1}\right)<y_{1}$ and $h^{n}\left(x_{2}\right)<y_{2}$ for all $n \in \mathbb{Z}$. Put $x=\min \left\{x_{1}, x_{2}\right\}$ and $y=\max \left\{y_{1}, y_{2}\right\}$. Then $g^{n}(x)<y$ and $h^{n}(x)<y$ for all $n \in \mathbb{Z}$; in particular, $y$ does not belong to neither $I_{g}(x)$ nor $I_{h}(x)$. Since $x$ belongs to both sets, we have either $I_{g}(x) \subset I_{h}(x)$ or $I_{h}(x) \subset I_{g}(x)$. Both cases being analogous, let us consider only the first one. Then for all $x^{\prime} \in I_{g}(x)$ we have $I_{h}\left(x^{\prime}\right) \subset I_{g}\left(x^{\prime}\right)=I_{g}(x)$. In particular, $h^{ \pm 1}\left(x^{\prime}\right)$ belongs to $I_{g}(x)$ for all $x^{\prime} \in I_{g}(x)$. Since the same holds for $g^{ \pm 1}\left(x^{\prime}\right)$, this easily implies that $(g h)^{n}(x) \in I_{g}(x)$ for all $n \in \mathbb{Z}$. As a consequence, $(g h)^{n}(x)<y$ for all $n \in \mathbb{Z}$, thus showing that $g h$ belongs to $\Gamma_{0}$.

Recall that for an ordered group $(\Gamma, \preceq)$, a convex jump is a pair $(G, H)$ of distinct $\preceq-$ convex subgroups such that $H$ is contained in $G$, and there is no $\preceq$-convex subgroup between them. The previously developed ideas lead naturally to the following result, which may be viewed as an extension of Conrad's theorem on the structure of convex subgroups for Conradian orderings [3, Theorem 4.1]. However, our proof follows ideas which are rather different from those of Conrad, and is much inspired from [12, Exercise 2.2.46].

Theorem 2.14 Let $(\Gamma, \preceq)$ be an ordered group, and let $(G, H)$ be a convex jump in $\Gamma$. Suppose that $G$ is a Conradian extension of $H$. Then $H$ is normal in $G$, and the ordering induced by $\preceq$ on the quotient $G / H$ is Archimedean (and hence order isomorphic to a subgroup of $(\mathbb{R},+)$, due to Hölder's theorem; see Botto Mura and Rhemtulla [1], Kopytov and Medvedev [8] and [12]).

Proof Let us consider the action of $G$ on the space of cosets $G / H$. Each element in $H$ fixes the coset $H$, and hence its action is not cofinal. By Proposition 2.13, if we show that the action of each element in $G \backslash H$ is cofinal, then this will give the normality of $H$ in $G$.

Now given $f \in G \backslash H$, let $G_{f}$ be the smallest convex subgroup of $G$ containing $H$ and $f$. We claim that $G_{f}$ coincides with the set $S_{f}=\left\{g \in G: f^{m} \prec g \prec\right.$ $f^{n}$ for some $m, n$ in $\left.\mathbb{Z}\right\}$. Indeed, $S_{f}$ is clearly a convex subset of $G$ containing $H$ and contained in $G_{f}$. Thus, for showing that $G_{f}=S_{f}$, we need to show that $S_{f}$ is a subgroup. For this, first note that, in the notation of the proof of Proposition 2.13, the conditions $g \in S_{f}$ and $I_{g}(H) \subset I_{f}(H)$ are equivalent. Therefore, for each $g \in S_{f}$ we have $I_{g^{-1}}(H)=I_{g}(H) \subset I_{f}(H)$, and thus $g^{-1} \in S_{f}$. Moreover, if $\bar{g}$ is another element in $S_{f}$, then $\bar{g} g H \in \bar{g}\left(I_{f}(H)\right)=I_{f}(H)$, and thus $I_{\bar{g} g}(H) \subset I_{f}(H)$. This means that $\bar{g} g$ belongs to $S_{f}$, thus concluding the proof that $S_{f}$ and $G_{f}$ coincide.

Each $f \in G \backslash H$ leads to a convex subgroup $G_{f}=S_{f}$ strictly containing $H$. Since $(G, H)$ is a convex jump, we necessarily have $S_{f}=G$. Given $g_{1} \prec g_{2}$ in $G$, choose 
$m_{1}, n_{2}$ in $\mathbb{Z}$ for which $f^{m_{1}} \prec g_{1}$ and $g_{2} \prec f^{n_{2}}$. Then we have $f^{n_{2}-m_{1}} g_{1} \succ$ $f^{n_{2}-m_{1}} f^{m_{1}}=f^{n_{2}} \succ g_{2}$, and hence $f^{n_{2}-m_{1}}\left(g_{1} H\right) \geq g_{2} H$. This easily implies that the action of $f$ is cofinal.

We have then showed that $H$ is normal in $G$. The left invariant total order on the space of cosets $G / H$ is therefore a group ordering. Moreover, given $f, g$ in $G$, with $f \notin H$, the previous argument shows that there exists $n \in \mathbb{Z}$ such that $f^{n} \succ g$, and thus $f^{n} H \succeq g H$. This is nothing but the Archimedean property for the induced ordering on $G / H$.

Corollary 2.15 Under the hypothesis of Theorem 2.14, up to multiplication by a positive real number, there exists a unique nontrivial group homomorphism $\tau: G \rightarrow \mathbb{R}$ such that $\operatorname{ker}(\tau)=H$ and $\tau(g)>0$ for every positive element $g \in G \backslash H$.

\section{On the approximation of a group ordering by its conju- gates}

\subsection{Describing the Conradian soul via crossings}

The Conradian soul $C_{\preceq}(\Gamma)$ of an ordered group $(\Gamma, \preceq)$ corresponds to the (unique) subgroup which is $\preceq$-convex, $\preceq-$ Conradian, and which is maximal among subgroups verifying these two properties simultaneously. This notion was introduced in [13], where a dynamical counterpart in the case of countable groups was given. To give an analogous characterization in the general case, we consider the set $S^{+}$formed by the elements $h \succ 1$ such that $h \preceq w$ for every crossing $(f, g, u, v, w)$ satisfying $1 \preceq u$. Analogously, we let $S^{-}$be the set formed by the elements $h \prec 1$ such that $w \preceq h$ for every crossing $(f, g, u, v, w)$ satisfying $v \preceq 1$. Finally, we let

$$
S=\{1\} \cup S^{+} \cup S^{-} \text {. }
$$

A priori, it is not clear that the set $S$ has a nice structure (for instance, it is not at all evident that it is actually a subgroup). However, this is largely shown by the theorem below.

Theorem 3.1 The Conradian soul of $(\Gamma, \preceq)$ coincides with the set $S$ above.

Before passing to the proof, we give four general lemmas on crossings for group orderings (note that the first three lemmas still apply to crossings for actions on totally ordered spaces). The first one allows us replacing the 'comparison' element $w$ by its 'images' under positive iterates of either $f$ or $g$. 
Lemma 3.2 If $(f, g, u, v, w)$ is a crossing, then $\left(f, g, u, v, g^{n} w\right)$ and $\left(f, g, u, v, f^{n} w\right)$ are also crossings for every $n \in \mathbb{N}$.

Proof We will only consider the first 5-tuple (the case of the second one is analogous). Recalling that $g w \succ w$, for every $n \in \mathbb{N}$ we have $u \prec w \prec g^{n} w$; moreover, $v \succ g^{M+n} u=$ $g^{n} g^{M} u \succ g^{n} w$. Hence, $u \prec g^{n} w \prec v$. On the other hand, $f^{N} v \prec w \prec g^{n} w$, while from $g^{M} u \succ w$ we get $g^{M+n} u \succ g^{n} w$.

Our second lemma allows replacing the 'limiting' elements $u$ and $v$ by more appropriate ones.

Lemma 3.3 Let $(f, g, u, v, w)$ be a crossing. If $f u \succ u$ (resp. $f u \prec u$ ) then $\left(f, g, f^{n} u, v, w\right)$ (resp. $\left.\left(f, g, f^{-n} u, v, w\right)\right)$ is also a crossing for every $n>0$. Analogously, if $g v \prec v$ (resp. $g v \succ v)$, then $\left(f, g, u, g^{n} v, w\right)\left(\operatorname{resp} .\left(f, g, u, g^{-n} v, w\right)\right)$ is also a crossing for every $n>0$.

Proof Let us only consider the first 5-tuple (the case of the second one is analogous). Suppose that $f u \succ u$ (the case $f u \prec u$ may be treated similarly). Then $f^{n} u \succ u$, which gives $g^{M} f^{n} u \succ g^{M} u \succ w$. To show that $f^{n} u \prec w$, assume by contradiction that $f^{n} u \succeq w$. Then $f^{n} u \succ f^{N} v$, which gives $u \succ f^{N-n} v$, which is absurd.

The third lemma relies on the dynamical insight of the crossing condition.

Lemma 3.4 If $(f, g, u, v, w)$ is a crossing, then $\left(h f h^{-1}, h g h^{-1}, h u, h v, h w\right)$ is also a crossing for every $h \in \Gamma$.

Proof The three conditions to be checked are nothing but the three conditions in the definition of crossing multiplied by $h$ by the left.

A direct application of the lemma above shows that, if $(f, g, u, v, w)$ is a crossing, then the 5-tuples $\left(f, f^{n} g f^{-n}, f^{n} u, f^{n} v, f^{n} w\right)$ and $\left(g^{n} f g^{-n}, g, g^{n} u, g^{n} v, g^{n} w\right)$ are also crossings for every $n \in \mathbb{N}$. This combined with Lemma 3.3 may be used to show the following.

Lemma 3.5 If $(f, g, u, v, w)$ is a crossing and $1 \preceq h_{1} \prec h_{2}$ are elements in $\Gamma$ such that $h_{1} \in S$ and $h_{2} \notin S$, then there exists a crossing $(\tilde{f}, \widetilde{g}, \tilde{u}, \tilde{v}, \tilde{w})$ such that $h_{1} \prec \tilde{u} \prec \widetilde{v} \prec h_{2}$. 
Proof Since $1 \prec h_{2} \notin S$, there must be a crossing $(f, g, u, v, w)$ such that $1 \preceq u \prec$ $w \prec h_{2}$. Let $N \in \mathbb{N}$ be such that $f^{N} v \prec w$. Denote by $(f, \bar{g}, \bar{u}, \bar{v}, \bar{w})$ the crossing $\left(f, f^{N} g f^{-N}, f^{N} u, f^{N} v, f^{N} w\right)$. Note that $\bar{v}=f^{N} v \prec w \prec h_{2}$. We claim that $h_{1} \preceq \bar{w}=f^{N} w$. Indeed, if $f^{N} u \succ u$ then $f^{n} u \succ 1$, and by the definition of $S$ we must have $h_{1} \preceq \bar{w}$. If $f^{N} u \prec u$, then we must have $f u \prec u$, so by Lemma 3.3 we know that $(f, \bar{g}, u, \bar{v}, \bar{w})$ is also a crossing, which allows still concluding that $h_{1} \preceq \bar{w}$.

Now for the crossing $(f, \bar{g}, \bar{u}, \bar{v}, \bar{w})$ there exists $M \in \mathbb{N}$ such that $\bar{w} \prec \bar{g}^{M} \bar{u}$. Let us consider the crossing $\left(\bar{g}^{M} f \bar{g}^{-M}, \bar{g}, \bar{g}^{M} \bar{u}, \bar{g}^{M} \bar{v}, \bar{g}^{M} \bar{w}\right)$. If $\bar{g}^{M} \bar{v} \prec \bar{v}$ then $\bar{g}^{M} \bar{v} \prec$ $h_{2}$, and we are done. If not, then it must be the case that $\bar{g} \bar{v} \succ \bar{v}$. By Lemma 3.3, $\left(\bar{g}^{M} f \bar{g}^{-M}, \bar{g}, \bar{g}^{M} \bar{u}, \bar{g}^{M} \bar{v}, \bar{w}\right)$ is still a crossing, and since $\bar{v} \prec h_{2}$, this concludes the proof.

Proof of Theorem 3.1 The proof is divided into several steps.

Claim 0 The set $S$ is convex.

This follows directly from the definition of $S$.

Claim 1 If $h$ belongs to $S$, then $h^{-1}$ also belongs to $S$.

Assume that $h \in S$ is positive and $h^{-1}$ does not belong to $S$. Then there exists a crossing $(f, g, u, v, w)$ so that $h^{-1} \prec w \prec v \preceq 1$.

We first note that, if $h^{-1} \preceq u$, then after conjugating by $h$ as in Lemma 3.4, we get a contradiction because $\left(h g h^{-1}, h f h^{-1}, h u, h v, h w\right)$ is a crossing with $1 \preceq h u$ and $h w \prec h v \preceq h$. To reduce the case $h^{-1} \succ u$ to this one, we first use Lemma 3.4 and we consider the crossing $\left(g^{M} f g^{-M}, g, g^{M} u, g^{M} v, g^{M} w\right)$. Since $h^{-1} \prec w \prec$ $g^{M} u \prec g^{M} w \prec g^{M} v$, if $g^{M} v \prec v$ then we are done. If not, Lemma 3.3 shows that $\left(g^{M} f g^{-M}, g, g^{M} u, g^{M} v, w\right)$ is also a crossing, which still allows concluding.

In the case where $h \in S$ is negative (that is, its inverse is positive) we proceed similarly but we conjugate by $f^{N}$ instead of $g^{M}$. Alternatively, since $1 \in S$ and $1 \prec h^{-1}$, if we suppose that $h^{-1} \notin S$ then Lemma 3.5 provides us with a crossing $(f, g, u, v, w)$ such that $1 \prec u \prec w \prec v \prec h^{-1}$, which gives a contradiction after conjugating by $h$.

Claim 2 If $h$ and $\bar{h}$ belong to $S$, then $h \bar{h}$ also belongs to $S$.

First we show that for every positive elements in $S$, their product still belongs to $S$. (Note that, by Claim 1, the same will be true for products of negative elements in $S$.) Indeed, suppose that $h, \bar{h}$ are positive elements, with $h \in S$ but $h \bar{h} \notin S$. Then, by Lemma 3.5 we may produce a crossing $(f, g, u, v, w)$ such that $h \prec u \prec v \prec h \bar{h}$. After conjugating by $h^{-1}$ we obtain the crossing $\left(h^{-1} f h, h^{-1} g h, h^{-1} u, h^{-1} v, h^{-1} w\right)$ satisfying $1 \prec h^{-1} u \prec h^{-1} w \prec \bar{h}$, which shows that $\bar{h} \notin S$. 
Now, if $h \prec 1 \prec \bar{h}$ then $h \prec h \bar{h}$. Hence, if $h \bar{h}$ is negative then the convexity of $S$ gives $h \bar{h} \in S$. If $h \bar{h}$ is positive, then $\bar{h}^{-1} h^{-1}$ is negative, and since $\bar{h}^{-1} \prec \bar{h}^{-1} h^{-1}$, the convexity gives again that $\bar{h}^{-1} h^{-1}$, and hence $h \bar{h}$, belongs to $S$. The remaining case $\bar{h} \prec 1 \prec h$ may be treated similarly.

Claim 3 The subgroup $S$ is Conradian.

In order to apply Theorem 2.4, we need to show that there are no crossings in $S$. Suppose by contradiction that $(f, g, u, v, w)$ is a crossing such that $f, g, u, v, w$ all belong to $S$. If $1 \preceq w$ then, by Lemma 3.4, we have that $\left(g^{n} f g^{-n}, g, g^{n} u, g^{n} v, g^{n} w\right)$ is a crossing. Taking $n=M$ so that $g^{M} u \succ w$, this gives a contradiction to the definition of $S$ because $1 \preceq w \prec g^{M} u \prec g^{M} w \prec g^{M} v \in S$. The case $w \preceq 1$ may be treated in an analogous way by conjugating by powers of $f$ instead of $g$.

Claim 4 The subgroup $S$ is maximal among $\preceq$-convex, $\preceq$-Conradian subgroups.

Indeed, if $C$ is a subgroup strictly containing $S$, then there is a positive $h$ in $C \backslash S$. By Lemma 3.5, there exists a crossing $(f, g, u, v, w)$ such that $1 \prec u \prec w \prec v \prec h$. If $C$ is convex, then $u, v, w$ belong to $C$. To conclude that $C$ is not Conradian, it suffices to show that $f$ and $g$ belong to $C$.

Since $1 \prec u$, we have either $1 \prec g \prec g u \prec v$ or $1 \prec g^{-1} \prec g^{-1} u \prec v$. In both cases, the convexity of $C$ implies that $g$ belongs to $C$. On the other hand, if $f$ is positive then from $f^{N} \prec f^{N} v \prec w$ we get $f \in C$, whereas in the case of a negative $f$ the inequality $1 \prec u$ gives $1 \prec f^{-1} \prec f^{-1} u \prec v$, which still shows that $f \in C$.

\subsection{Approximation of group orderings: the role of the Conradian soul}

For a (left) orderable group $\Gamma$, we denote by $\mathcal{L O}(\Gamma)$ the set of all orderings on $\Gamma$. This space carries the topology having as a subbasis the family of sets $U_{f}=\{\preceq: f \succ 1\}$, where $f \neq 1$. Endowed with this topology, $\mathcal{L O}(\Gamma)$ is called the space of (left) orderings of the group $\Gamma$.

Remark 3.6 As shown in [12], a simple application of Tychonov's theorem shows that $\mathcal{L O}(\Gamma)$ is always compact. Moreover, the ' $n=2$ ' property from Remark 2.5 implies that the subset of Conradian orderings is closed therein (and hence compact). A more dynamical argument for showing this consists in noticing that the condition that $(f, g, u, v, w)$ is a reinforced crossing for prescribed $M, N$ is clearly open in $\mathcal{L O}(\Gamma)$ (see Remark 2.6).

The positive cone of an ordering $\preceq$ in $\mathcal{L O}(\Gamma)$ is the set $P$ of its positive elements. Because of the left invariance, $P$ completely determines $\preceq$. The conjugate of $\preceq$ by 
$h \in \Gamma$ is the ordering $\preceq_{h}$ having positive cone $h P h^{-1}$. In other words, $g \succ_{h} 1$ holds if and only if $h g h^{-1} \succ 1$. We will say that $\preceq$ may be approximated by its conjugates if it is an accumulation point of its set of conjugates.

Theorem 3.7 If the Conradian soul of an (infinite) ordered group $(\Gamma, \preceq)$ is trivial, then $\preceq$ may be approximated by its conjugates.

Proof Let $f_{1} \prec f_{2} \prec \cdots \prec f_{k}$ be finitely many positive elements in $\Gamma$. We need to show that there exists a conjugate of $\preceq$ which is different from $\preceq$ but for which all the $f_{i}$ 's are still positive. Since $1 \in C_{\preceq}(\Gamma)$ and $f_{1} \notin C_{\preceq}(\Gamma)$, Theorem 3.1 and Lemma 3.5 imply that there is a crossing $(f, g, u, v, w)$ such that $1 \prec u \prec v \prec f_{1}$. Let $M, N$ in $\mathbb{N}$ be such that $f^{N} v \prec w \prec g^{M} u$. We claim that $1 \prec_{v^{-1}} f_{i}$ and $1 \prec_{w^{-1}} f_{i}$ for $1 \leq i \leq k$, but $g^{M} f^{N} \prec_{v^{-1}} 1$ and $g^{M} f^{N} \succ_{w^{-1}} 1$. Indeed, since $1 \prec v \prec f_{i}$, we have $v \prec f_{i} \prec f_{i} v$, thus $1 \prec v^{-1} f_{i} v$. By definition, this means that $f_{i} \succ_{v^{-1}} 1$. The inequality $f_{i} \succ_{w^{-1}} 1$ is proved similarly. Now note that $g^{M} f^{N} v \prec g^{M} w \prec v$, and so $g^{M} f^{N} \prec_{v^{-1}}$ 1. Finally, from $g^{M} f^{N} w \succ g^{M} u \succ w$ we get $g^{M} f^{N} \succ_{w^{-1}} 1$.

Now the preceding relations imply that the $f_{i}$ 's are still positive for both $\preceq_{v^{-1}}$ and $\preceq w^{-1}$, but at least one of these orderings is different from $\preceq$. This concludes the proof.

Based on the work of Linnell [9], it is shown in [13, Proposition 4.1] that no Conradian ordering is an isolated point of the space of orderings of a group having infinitely many orderings. Together with Theorem 3.7, this shows the next proposition by means of the convex extension procedure (see Remark 2.12).

Proposition 3.8 Let $\Gamma$ be an orderable group. If $\preceq$ is an isolated point of $\mathcal{L O}(\Gamma)$, then its Conradian soul is non-trivial and has only finitely many orderings.

As a consequence of a nice theorem of Tararin, the number of orderings on an orderable group having only finitely many orderings is a power of 2; moreover, all of these orderings are necessarily Conradian (see Kopytov and Medvedev [8] and [12]). By the preceding proposition, if $\preceq$ is an isolated point in the space of orderings $\mathcal{L O}(\Gamma)$, then its Conradian soul admits $2^{n}$ different orderings for some $n \geq 1$, all of them Conradian. Let $\left\{\preceq_{1}, \preceq_{2}, \ldots, \preceq_{2^{n}}\right\}$ be these orderings, where $\preceq_{1}$ is the restriction of $\preceq$ to its Conradian soul. Since $C_{\preceq}(\Gamma)$ is $\preceq$-convex, each $\preceq_{j}$ induces an ordering $\preceq^{j}$ on $\Gamma$, namely the convex extension of $\preceq_{j}$ by $\preceq$. (Note that $\preceq^{1}$ coincides with $\preceq$.) All the orderings $\preceq^{j}$ share the same Conradian soul [13, Lemma 3.37]. Assume throughout that $\preceq$ is not Conradian. 
Theorem 3.9 With the notation above, at least one of the orderings $\preceq^{j}$ is an accumulation point of the set of conjugates of $\preceq$.

Corollary 3.10 At least one of the orderings $\preceq^{j}$ is approximated by its conjugates.

Proof Asumming Theorem 3.9, we have $\preceq^{k} \in \operatorname{acc}\left(\operatorname{orb}\left(\swarrow^{1}\right)\right)$ for some $k \in\left\{1, \ldots, 2^{n}\right\}$, where orb $\left(\preceq^{1}\right)$ stands for the orbit of $\preceq^{1}$ under the conjugacy action of $\Gamma$, and $\operatorname{acc}\left(\operatorname{orb}\left(\swarrow^{1}\right)\right)$ denotes the set of its accumulation points. Theorem 3.9 applied to this $\preceq^{k}$ instead of $\preceq$ shows the existence of $k^{\prime} \in\left\{1, \ldots, 2^{n}\right\}$ so that $\preceq^{k^{\prime}} \in \operatorname{acc}\left(\operatorname{orb}\left(\preceq^{k}\right)\right)$, and hence $\preceq^{k^{\prime}} \in \operatorname{acc}\left(\operatorname{orb}\left(\preceq^{1}\right)\right)$. If $k^{\prime}$ equals either 1 or $k$ then we are done; if not, we continue arguing in this way... In at most $2^{n}$ steps we will find an index $j$ such that $\preceq^{j} \in \operatorname{acc}\left(\operatorname{orb}\left(\preceq^{j}\right)\right)$.

Theorem 3.9 will follow from the next proposition.

Proposition 3.11 Given an arbitrary finite family $\mathcal{G}$ of $\preceq$-positive elements in $\Gamma$, there exists $h \in \Gamma$ and $1 \prec \bar{h} \notin C_{\preceq}(\Gamma)$ such that $1 \prec h^{-1} f h \notin C_{\preceq}(\Gamma)$ for all $f \in \mathcal{G} \backslash C_{\preceq}(\Gamma)$, but $1 \succ h^{-1} \bar{h} h \notin C_{\preceq}(\Gamma)$.

Proof of Theorem 3.9 from Proposition 3.11 Let us consider the directed set formed by the finite sets $\mathcal{G}$ of $\preceq$-positive elements. For each such a $\mathcal{G}$, let $h_{\mathcal{G}}$ and $\bar{h}_{\mathcal{G}}$ be the elements in $\Gamma$ provided by Proposition 3.11. After passing to subnets of $\left(h_{\mathcal{G}}\right)$ and $\left(\bar{h}_{\mathcal{G}}\right)$ if necessary, we may assume that the restrictions of $\preceq_{h_{\mathcal{G}}^{-1}}$ to $C_{\preceq}(\Gamma)$ all coincide with a single $\preceq_{j}$. Now the properties of $h_{\mathcal{G}}$ and $\bar{h}_{\mathcal{G}}$ imply

- $f \succ^{j} 1$ and $f\left(\succ^{j}\right)_{h_{\mathcal{G}}^{-1}} 1$ for all $f \in \mathcal{G} \backslash C_{\preceq}(\Gamma)$,

- $\bar{h}_{\mathcal{G}} \succ 1$, but $\bar{h}_{\mathcal{G}}\left(\prec^{j}\right)_{h_{\mathcal{G}}^{-1}} \prec 1$.

This clearly shows the Theorem.

For the proof of Proposition 3.11 we will use three general lemmas.

Lemma 3.12 For every $1 \prec c \notin C_{\preceq}(\Gamma)$ there is a crossing $(f, g, u, v, w)$ such that $u, v, w$ do not belong to $C_{\leq}(\Gamma)$ and $1 \prec u \prec w \prec v \prec c$.

Proof By Theorem 3.1 and Lemma 3.5, for every $1 \preceq s \in C_{\preceq}(\Gamma)$ there exists a crossing $(f, g, u, v, w)$ such that $s \prec u \prec w \prec v \prec c$. Clearly, $v$ does not belong to $C_{\preceq}(\Gamma)$. The element $w$ is also outside $C_{\preceq}(\Gamma)$, since in the other case the element $a=w^{2}$ would satisfy $w \prec a \in C_{\preceq}(\Gamma)$, which is absurd. Taking $M>0$ so that 
$g^{M} u \succ w$, this gives $g^{M} u \notin C_{\preceq}(\Gamma), g^{M} w \notin C_{\preceq}(\Gamma)$, and $g^{M} v \notin C_{\preceq}(\Gamma)$. Consider the crossing $\left(g^{M} f g^{-M}, g, g^{M} u, g^{M} v, g^{M} w\right)$. If $g^{M} v \prec v$, then we are done. If not, then $g v \succ v$, and Lemma 3.3 ensures that $\left(g^{M} f g^{-M}, g, g^{M} u, v, g^{M} w\right)$ is also a crossing, which still allows concluding.

Lemma 3.13 Given $1 \prec c \notin C_{\preceq}(\Gamma)$ there exists $1 \prec a \notin C_{\preceq}(\Gamma)$ (with $a \prec c$ ) such that, for all $1 \preceq b \preceq a$ and all $\bar{c} \succeq c$, one has $1 \prec b^{-1} \bar{c} b \notin C_{\preceq}(\Gamma)$.

Proof Let us consider the crossing $(f, g, u, v, w)$ such that $1 \prec u \prec w \prec v \prec c$ and such that $u, v, w$ do not belong to $C_{\preceq}(\Gamma)$. We affirm that the lemma holds for $a=u$ (actually, it holds for $a=w$, but the proof is slightly more complicated). Indeed, if $1 \preceq b \preceq u$, then from $b \preceq u \prec v \prec \bar{c}$ we get $1 \preceq b^{-1} u \prec b^{-1} v \prec b^{-1} \bar{c}$, and thus the crossing $\left(b^{-1} f b, b^{-1} g b, b^{-1} u, b^{-1} v, b^{-1} w\right)$ shows that $b^{-1} \bar{c} \notin C_{\preceq}(\Gamma)$. Since $1 \preceq b$, we conclude that $1 \prec b^{-1} \bar{c} \preceq b^{-1} \bar{c} b$, and the convexity of $S$ implies that $b^{-1} \bar{c} b \notin C_{\preceq}(\Gamma)$.

Lemma 3.14 For every $g \in \Gamma$ the set $g C_{\preceq}(\Gamma)$ is convex. Moreover, for every crossing $(f, g, u, v, w)$ one has $u C_{\preceq}(\Gamma)<w C_{\preceq}(\Gamma)<v C_{\preceq}(\Gamma)$, in the sense that $u h_{1} \prec w h_{2} \prec v h_{3}$ for all $h_{1}, h_{2}, h_{3}$ in $C_{\preceq}(\Gamma)$ (see Example 2.11).

Proof The verification of the convexity of $g C_{\preceq}(\Gamma)$ is straightforward. Now suppose that $u h_{1} \succ w h_{2}$ for some $h_{1}, h_{2}$ in $C_{\preceq}(\Gamma)$. Then since $u \prec w$, the convexity of both left classes $u C_{\preceq}(\Gamma)$ and $w C_{\preceq}(\Gamma)$ gives the equality between them. In particular, there exists $h \in C_{\preceq}(\Gamma)$ such that $u h=w$. Note that such an $h$ must be positive, so that $1 \prec h=u^{-1} w$. But since $\left(u^{-1} f u, u^{-1} g u, 1, u^{-1} v, u^{-1} w\right)$ is a crossing, this contradicts the definition of $C_{\preceq}(\Gamma)$. Showing that $w C_{\preceq}(\Gamma) \prec v C_{\preceq}(\Gamma)$ is similar.

Proof of Proposition 3.11 Let us label the elements of $\mathcal{G}=\left\{f_{1}, \ldots, f_{r}\right\}$ so that $f_{1} \prec \cdots \prec f_{r}$, and let $k$ be such that $f_{k-1} \in C_{\preceq}(\Gamma)$ but $f_{k} \notin C_{\preceq}(\Gamma)$. Recall that, by Lemma 3.13, there exists $1 \prec a \notin C_{\preceq}(\Gamma)$ such that, for every $1 \preceq b \preceq a$, one has $1 \prec b^{-1} f_{k+j} b \notin C_{\preceq}(\Gamma)$ for all $j \geq 0$. We fix a crossing $(f, g, u, v, w)$ such that $1 \prec u \prec v \prec a$ and $u \notin C_{\preceq}(\Gamma)$. Note that the conjugacy by $w^{-1}$ gives the crossing $\left(w^{-1} f w, w^{-1} g w, w^{-1} u, w^{-1} v, 1\right)$.

Case 1 One has $w^{-1} v \preceq a$.

In this case, the proposition holds for $h=w^{-1} v$ and $\bar{h}=w^{-1} g^{M+1} f^{N} w$. To show this, first note than neither $w^{-1} g w$ nor $w^{-1} f w$ belong to $C_{\preceq}(\Gamma)$. Indeed, this follows from the convexity of $C_{\preceq}(\Gamma)$ and the inequalities $w^{-1} g^{-M} w \prec w^{-1} u \notin C_{\preceq}(\Gamma)$ and $w^{-1} f^{-N} w \succ w^{-1} v \notin C_{\preceq}(\Gamma)$. We also have $1 \prec w^{-1} g^{M} f^{N} w$, and hence 
$1 \prec w^{-1} g w \prec w^{-1} g^{M+1} f^{N} w$, which shows that $\bar{h} \notin C_{\preceq}(\Gamma)$. On the other hand, the inequality $w^{-1} g^{M+1} f^{N} w\left(w^{-1} v\right) \prec w^{-1} v$ reads as $h^{-1} \bar{h} h \prec 1$.

Finally, Lemma 3.2 applied to the crossing $\left(w^{-1} f w, w^{-1} g w, w^{-1} u, w^{-1} v, 1\right)$ shows that $\left(w^{-1} f w, w^{-1} g w, w^{-1} u, w^{-1} v, w^{-1} g^{M+n} f^{N} w\right)$ is a crossing for every $n>$ 0 . For $n \geq M$ we have $w^{-1} g^{M+1} f^{N} w\left(w^{-1} v\right) \prec w^{-1} g^{M+n} f^{N} w$, and since $w^{-1} g^{M+n} f^{N} w \prec w^{-1} v$, Lemma 3.14 easily implies that

$$
w^{-1} g^{M+1} f^{N} w\left(w^{-1} v\right) C_{\preceq}(\Gamma) \prec w^{-1} v C_{\preceq}(\Gamma),
$$

that is, $h^{-1} \bar{h} h \notin C_{\preceq}(\Gamma)$.

Case 2 One has $a \prec w^{-1} v$, but $w^{-1} g^{m} w \preceq a$ for all $m>0$.

We claim that, in this case, the proposition holds for $h=a$ and $\bar{h}=w^{-1} g^{M+1} f^{N} w$. This may be checked in the very same way as in Case 1 by noticing that, if $a \prec w^{-1} v$ but $w^{-1} g^{m} w \succeq a$ for all $m>0$, then $\left(w^{-1} f w, w^{-1} g w, w^{-1} u, a, 1\right)$ is a crossing.

Case 3 One has $a \prec w^{-1} v$ and $w^{-1} g^{m} w \succ a$ for some $m>0$. (Note that the first condition follows from the second one.)

We claim that, in this case, the proposition holds for $h=a$ and $\bar{h}=w \notin C_{\preceq}(\Gamma)$. Indeed, we have $g^{m} w \succ h a$ (and $w \prec h a$ ), and since $g^{m} w \prec v \prec a$, we have $w a \prec a$, which means that $h^{-1} \bar{h} h \prec 1$. Finally, from Lemmas 3.2 and 3.14 we get $w a C_{\preceq}(\Gamma) \preceq$ $g^{m} w C_{\preceq}(\Gamma) \prec v C_{\preceq}(\Gamma) \preceq a C_{\preceq}(\Gamma)$. This implies that $a^{-1} w a C_{\preceq}(\Gamma) \prec C_{\preceq}(\Gamma)$, which means that $h^{-1} \bar{h} h \notin C_{\preceq}(\Gamma)$.

\section{Finitely many or uncountably many group orderings}

The goal of this final short section is to use the previously developed ideas to show the following result.

Theorem 4.1 If the space of orderings of an orderable group is infinite, then it is uncountable.

Proof Let us fix an ordering $\preceq$ on an orderable group $\Gamma$. We need to analize two different cases.

Case 1 The Conradian soul of $C_{\preceq}(\Gamma)$ is non-trivial and has infinitely many orderings. This case was settled in [13, Proposition 4.1] using ideas going back to Zenkov [15] and Tararin (see Kopytov and Medvedev [8]).

Case 2 The Conradian soul of $C_{\preceq}(\Gamma)$ has only finitely many orderings. 
If $\preceq$ is Conradian, then $\Gamma=C_{\preceq}(\Gamma)$ has finitely many orderings. If not, then Theorems 3.7 and 3.9 imply that there exists an ordering $\preceq^{*}$ on $\Gamma$ which is an accumulation point of its conjugates. The closure in $\mathcal{L O}(\Gamma)$ of the set of conjugates of $\preceq^{*}$ is then a compact set without isolated points. By a well-known fact in General Topology, such a set must be uncountable. Therefore, $\Gamma$ admits uncountably many orderings.

\section{Appendix A An exotic ordering of $F_{2}$}

We construct an ordering of $F_{2}$ which is not an accumulation point of its conjugates in $\mathcal{L O}\left(F_{2}\right)$ and whose Conradian soul is isomorphic to $\mathbb{Z}$. This ordering is realized as the restriction of the Dubrovina-Dubrovin ordering of $B_{3}$ to an appropriate free subgroup of $B_{3}$.

We begin by defining the Dehornoy ordering of the braid groups (also known as the 'standard' ordering), whose positive cone we shall denote $P_{D}$ (see Dehornoy [5] and Dehornoy-Dynnikov-Rolfsen-Wiest [6]). Recall that for each integer $n \geq 2$, the Artin braid group $B_{n}$ is the group generated by $\sigma_{1}, \sigma_{2}, \ldots, \sigma_{n-1}$, subject to the relations

$$
\sigma_{i} \sigma_{j}=\sigma_{j} \sigma_{i} \text { if }|i-j|>1, \quad \sigma_{i} \sigma_{j} \sigma_{i}=\sigma_{j} \sigma_{i} \sigma_{j} \text { if }|i-j|=1 .
$$

Definition A.1 Let $w$ be a word in the generators $\sigma_{i}, \cdots, \sigma_{n-1}$ (so no $\sigma_{j}$ occurs for $j<i$ ). Then $w$ is said to be: $i$-positive if the generator $\sigma_{i}$ occurs in $w$ with only positive exponents, $i$-negative if $\sigma_{i}$ occurs with only negative exponents, and $i$-neutral if $\sigma_{i}$ does not occur in $w$.

We then define the positive cone of the Dehornoy ordering as

Definition A.2 The positive cone $P_{D} \subset B_{n}$ of the Dehornoy ordering is the set

$$
P_{D}=\left\{\beta \in B_{n}: \beta \text { is } i \text {-positive for some } i \leq n-1\right\} \text {. }
$$

An extremely important property of this ordering is that the conjugate $\beta \sigma_{k} \beta^{-1}$ is always $i$-positive for some $i$, for every generator $\sigma_{k}$ in $B_{n}$ and any braid $\beta \in B_{n}$. This property is referred to as the subword property [6].

There is also a second ordering of interest, discovered by the authors of [7], whose positive cone we shall denote by $P_{D D}$. Denote by $P_{i} \subset B_{n}$ the set of all $i$-positive braids. Note that the set of all $i$-negative braids is simply $P_{i}^{-1}$.

Definition A.3 The positive cone $P_{D D} \subset B_{n}$ is the set

$$
P_{D D}=P_{1} \cup P_{2}^{-1} \cup \cdots \cup P_{n-1}^{(-1)^{n}} \text {. }
$$


As is well-known, the subgroup of $B_{3}$ generated by the elements $\sigma_{1}^{2}, \sigma_{2}^{2}$ is isomorphic to $F_{2}$, the free group on two generators (see also Crisp and Paris [4]). Thus we may consider $F_{2}$ to be the subgroup of $B_{3}$ generated by $\sigma_{1}^{2}$ and $\sigma_{2}^{2}$, and define a positive cone $P$ in $F_{2}$ by $P=P_{D D} \cap F_{2}$. Note that any element of $F_{2}$ must always be represented by a braid word having even total exponent, and that the ordering $\preceq_{C}$ of $F_{2}$ asssociated to the positive cone $P$ is simply the restriction of the $P_{D D}$ ordering to the subgroup $\left\langle\sigma_{1}^{2}, \sigma_{2}^{2}\right\rangle$.

Proposition A.4 The ordering $\preceq_{C}$ is not an accumulation point of its conjugates in $\mathcal{L O}\left(F_{2}\right)$. Specifically, no conjugates of $\preceq_{C}$ distinct from $\preceq_{C}$ lie inside the open set $U_{\sigma_{2}^{-2}} \subset \mathcal{L O}\left(F_{2}\right)$.

Proof Let $\beta \in F_{2} \subset B_{3}$ be given, and consider the positive cone $\beta P \beta^{-1}$. To prove the claim, it suffices to show that $\sigma_{2}^{-2} \in \beta P \beta^{-1}$ implies $\beta P \beta^{-1}=P$.

First, observe that conjugation of $P$ by any even power of $\sigma_{2}$ does not change $P$ : this follows from the fact that $\sigma_{2}^{-2}$ is the least positive element in the associated ordering $\preceq_{C}$ of $F_{2}$. Indeed, for any element $g \in P$, we have $\sigma_{2}^{-2} \preceq_{C} g$, so that $\sigma_{2}^{2} g \succeq_{C} 1$, and hence $\sigma_{2}^{2} g \sigma_{2}^{-2} \succ_{C} 1$, that is, $\sigma_{2}^{2} g \sigma_{2}^{-2} \in P$.

Now with $\sigma_{2}^{-2} \in \beta P \beta^{-1}$, in particular we must have $\beta^{-1} \sigma_{2}^{-2} \beta \in P$. Since $P$ consists of those elements of $F_{2}$ that are either 1-positive or 2 -negative, by the subword property, we know that $\beta^{-1} \sigma_{2}^{-2} \beta$ is not 1 -positive, and so must be 2 -negative. Therefore $\beta^{-1} \sigma_{2}^{-2} \beta=\sigma_{2}^{k}$ for some $k<0$, and in fact, by considering total exponents we see that $\beta^{-1} \sigma_{2}^{-2} \beta=\sigma_{2}^{-2}$.

Recall that we are working in $F_{2} \subset B_{3}$, so $\beta$ cannot commute with $\sigma_{2}^{-2}$ unless $\beta$ itself is an even power of $\sigma_{2}$ (the power must be even since $\beta \in F_{2}=\left\langle\sigma_{1}^{2}, \sigma_{2}^{2}\right\rangle$ ). Therefore $\sigma_{2}^{-2} \in \beta P \beta^{-1}$ implies $\beta=\sigma_{2}^{2 k}$, so that $\beta P \beta^{-1}=P$.

Next, we show that the only non-trivial convex subgroup in the ordering $\preceq_{C}$ of $F_{2}$ defined by $P$ is $\left\langle\sigma_{2}^{-2}\right\rangle$, the infinite cyclic group generated by the least positive element $\sigma_{2}^{-2}$. In particular, this shows that the Conradian soul of the ordering $\preceq_{C}$ of $F_{2}$ is isomorphic to $\mathbb{Z}$.

Theorem A.5 Suppose that $S$ is a subgroup of $F_{2}$ that is convex in the ordering $\preceq_{C}$. If $S$ properly contains $\left\langle\sigma_{2}^{-2}\right\rangle$, then $S=F_{2}$.

Proof Let $S$ be a convex subgroup properly containing $\left\langle\sigma_{2}^{-2}\right\rangle$. As the containment is proper, $S$ must contain a 1 -positive braid $\beta$. Suppose that $\beta$ is represented by 
the 1 -positive braid word $\sigma_{2}^{k} \sigma_{1} w$ where $k \in \mathbb{Z}$, and $w$ is a 1 -positive, 1 -neutral or empty braid word. Left multiplying by an appropriate power of $\sigma_{2}^{2}$, we may produce a new 1-positive braid $\beta^{\prime}=\sigma_{2}^{2 l} \beta$ in $S$ that is represented by a 1 -positive braid word of the form $\sigma_{2}^{k^{\prime}} \sigma_{1} w$, where $k^{\prime}=2 l+k>0$. Note that $\beta^{\prime} \in S$, since both $\beta$ and $\sigma_{2}^{2}$ lie in $S$.

Consider the braid represented by the word $\sigma_{1}^{-2} \sigma_{2}^{k^{\prime}} \sigma_{1} w$. For any $m$, we have $\sigma_{1}^{-1} \sigma_{2}^{m} \sigma_{1}=\sigma_{2} \sigma_{1}^{m} \sigma_{2}^{-1}$, so that we compute

$$
\sigma_{1}^{-2} \sigma_{2}^{k^{\prime}} \sigma_{1} w=\sigma_{1}^{-1} \sigma_{2} \sigma_{1}^{k^{\prime}} \sigma_{2}^{-1} w=\sigma_{1}^{-1} \sigma_{2} \sigma_{1} \sigma_{1}^{k^{\prime}-1} \sigma_{2}^{-1} w=\sigma_{2} \sigma_{1} \sigma_{2}^{-1} \sigma_{1}^{k^{\prime}-1} \sigma_{2}^{-1} w,
$$

and since $k^{\prime}>0$ and $w$ is a 1-positive, 1-neutral or empty word, we see that $\sigma_{1}^{-2} \sigma_{2}^{k^{\prime}} \sigma_{1} w$ represents a 1 -positive braid. Therefore, in the ordering $\preceq_{C}$ of $F_{2}$, we have

$$
1 \prec_{C} \sigma_{1}^{-2} \sigma_{2}^{k^{\prime}} \sigma_{1} w \Rightarrow \sigma_{1}^{2} \prec_{C} \sigma_{2}^{k^{\prime}} \sigma_{1} w=\beta^{\prime} .
$$

Since $1 \prec_{C} \sigma_{1}^{2}$ and $\beta^{\prime} \in S$, we conclude that $\sigma_{1}^{2} \in S$. But then $S$ contains both $\sigma_{2}^{2}$ and $\sigma_{1}^{2}$, the generators of $F_{2}$, so that $S=F_{2}$.

Remark A.6 From the work of McCleary [10] and from [13], it is known that the ordering $\preceq_{C}$ is not an isolated point in $\mathcal{L O}\left(F_{2}\right)$, but no method of constructing a sequence converging to $\preceq_{C}$ is given therein. Given an ordering $\preceq$ in $\mathcal{L O}\left(F_{2}\right)$, the known methods for constructing a sequence converging to $\preceq$ involve either approximation using the conjugates of $\preceq$, or approximation by modifying the ordering on the convex jumps in the Conradian soul of $\preceq$. The results of this Appendix show that neither of these methods is sufficient for constructing a sequence of orderings converging to $\preceq_{C}$.

\section{References}

[1] R Botto Mura, A Rhemtulla, Orderable groups, Lecture Notes in Pure and Applied Mathematics 27, Marcel Dekker, New York (1977) MR0491396

[2] A Clay, Isolated points in the space of left orderings of a group, preprint (2008)

[3] P Conrad, Right-ordered groups, Michigan Math. J. 6 (1959) 267-275 MR0106954

[4] J Crisp, L Paris, The solution to a conjecture of Tits on the subgroup generated by the squares of the generators of an Artin group, Invent. Math. 145 (2001) 19-36 MR1839284

[5] P Dehornoy, Braid groups and left distributive operations, Trans. Amer. Math. Soc. 345 (1994) 115-150 MR1214782

[6] P Dehornoy, I Dynnikov, D Rolfsen, B Wiest, Why are braids orderable?, Panoramas et Synthèses 14, Société Mathématique de France, Paris (2002) MR1988550 
[7] T V Dubrovina, N I Dubrovin, On braid groups, Mat. Sb. 192 (2001) 53-64 MR1859702

[8] V M Kopytov, N Y Medvedev, Right-ordered groups, Siberian School of Algebra and Logic, Consultants Bureau, New York (1996) MR1393199

[9] P A Linnell, Noncommutative localization in group rings, from: "Non-commutative localization in algebra and topology", London Math. Soc. Lecture Note Ser. 330, Cambridge Univ. Press, Cambridge (2006) 40-59 MR2222481

[10] S H McCleary, An even better representation for free lattice-ordered groups, Trans. Amer. Math. Soc. 290 (1985) 81-100 MR787956

[11] D W Morris, Amenable groups that act on the line, Algebr. Geom. Topol. 6 (2006) 2509-2518 MR2286034

[12] A Navas, Grupos de difeomorfismos del círculo, Ensaios Matemáticos 13, Sociedade Brasileira de Matemática, Rio de Janeiro (2007) MR2394157

[13] A Navas, On the dynamics of left-orderable groups, preprint (2007)

[14] A S Sikora, Topology on the spaces of orderings of groups, Bull. London Math. Soc. 36 (2004) 519-526 MR2069015

[15] A V Zenkov, On groups with an infinite set of right orders, Sibirsk. Mat. Zh. 38 (1997) 90-92, ii MR1446675

Departamento de Matemática y Ciencia de la Computación, Facultad de Ciencia Universidad de Santiago de Chile, Alameda 3363, Estación Central, Santiago, Chile Departamento de Matemáticas, Facultad de Ciencia Universidad de Chile, Las Palmeras 3425, Ñuñoa, Santiago, Chile

\section{Adam Clay}

Department of Mathematics, University of British Columbia Vancouver, British Columbia, Canada, V6T $1 Z 2$ andres.navas@usach.cl, cristobalrivas@u.uchile.cl, aclay@math.ubc.ca http://www.math.ubc.ca/ aclay/

Received: 3 March 2009 Revised: 28 August 2009 